\title{
ESA Derived ROTEM parameters in End-Stage Liver Disease. An insight into cirrhotic coagulopathy.
}

\section{Popescu M., Tomescu D.}

Carol Davila University of Medicine and Pharmacy, Fundeni Clinical Hospital, Anesthesia and Critical Care Dept III., Bucharest, Romania

\section{INTRODUCTION.}

The re-balanced approach on hemostasis in End-Stage Liver Disease (ESLD) has changed the way we investigate and treat cirrhotic coagulopathy. Viscoelasting tests have become widely used in such patients, especially during the perioperative period. We hypothesize that derived ROTEM parameters may offer new information about the pathophysiological mechanisms of cirrhotic coagulopathy.

\section{METHODS.}

162 cirrhotic patients were prospectively included during a two year period: March 2013- March 2015. Collected data were: demographic data, etiology and severity of ESLD, liver functional tests, standard coagulation tests, platelet count and ROTEM parameters. Four ROTEM tests (ExTEM, InTEM, FibTEM, ApTEM) were performed on each patient and both standard (clotting time-CT, clot formation time-CFT, maximum clot firmness-MCF) and derived (thrombin potential index-TPI, maximum velocity of clot formationMaxV, time to MaxV-MaxVt, area under the curve-AUC and maximum clot elasticity-MCE) parameters were recorded.

\section{RESULTS.}

Patients mean age was $54 \pm 12.6$ years and mean MELD score was 19.19 \pm 6.27 . INTEM CFT strongly correlated with AUC $(p=0.003), \operatorname{MCE}(p=0.001)$ and TPI $(p=0.001)$. ExTEM CFT correlated with TPI $(\mathrm{p}=0.003)$ and we determined a cut-off value of 20 for ExTEM TPI below which ExTEM CFT rose exponentially. Platelet count correlated with ExTEM MCF $(p=0.008)$, ExTEM TPI $(p=0.000), \operatorname{MaxV}(p=0.045)$ and $\operatorname{MCE}(p=0.000)$. We determined a cut-off value of 60000 for platelets below which ExTEM clot formation became significantly impaired and a cut-off value of 70000 below which clot elasticity significantly decreased.

\section{CONCLUSIONS.}

Derived thromboelastometric parameters can offer a complex pathophysiological explanation of cirrhotic coagulopathy. Our results demonstrate the central role of platelets in determining clot strength and elasticity. The use of such parameters in clinical practice remains to be further evaluated.

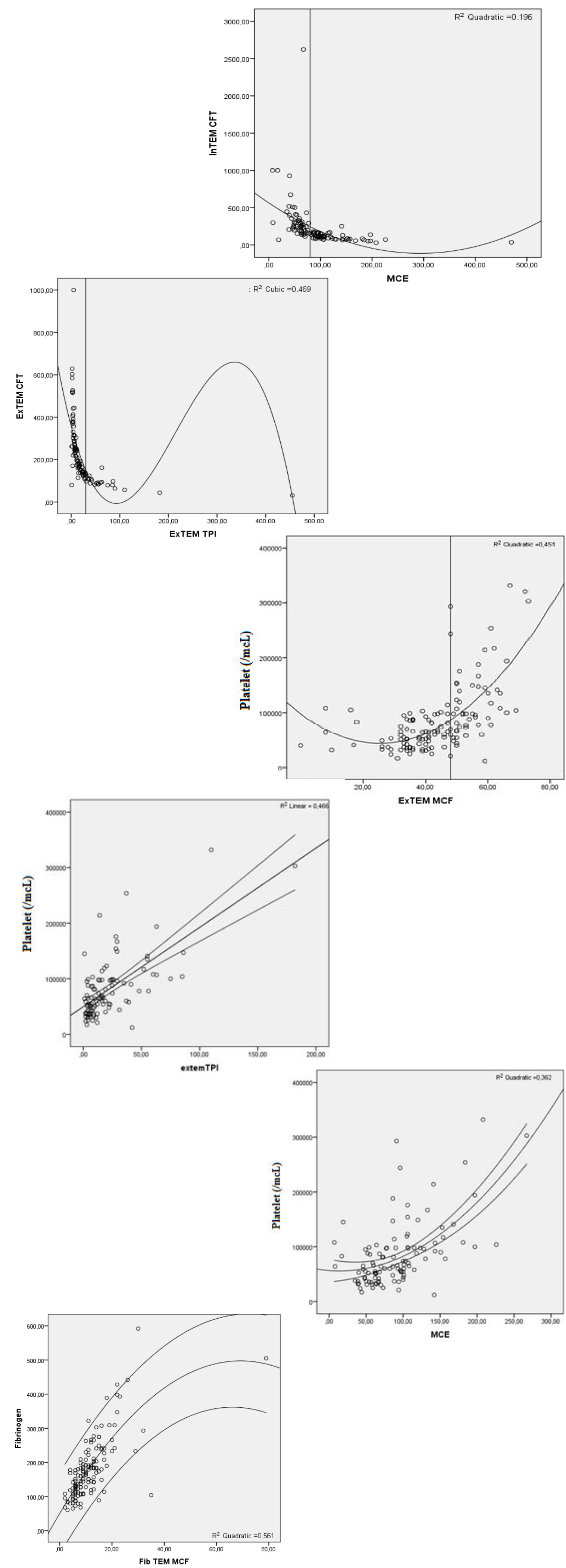

